\title{
Effect of lubricant molecular rheology on formation and shear of ultra-thin surface films
}

\author{
W. W. F. Chong ${ }^{1}$, M. Teodorescu ${ }^{1}$ and H. Rahnejat ${ }^{2}$ \\ ${ }^{1}$ School of Engineering, Cranfield University, Cranfield, UK \\ ${ }^{2}$ Wolfson School of Mechanical \& Manufacturing Engineering, Loughborough \\ University, Loughborough, UK \\ E-mail: m.s.teodorescu@cranfield.ac.uk
}

\begin{abstract}
Physics of molecularly thin fluid films, formed between surface features at close range is investigated. It is found that the interplay between discrete lubricant drainage from such contacts and localised contact deflection plays an important role both on the load carrying capacity of these asperity level conjunctions as well as on friction. Small spherical molecules tend to solvate near assumed smooth surfaces of asperities at nano-scale. Their discrete drainage at steadily decreasing gaps adds to the viscous friction of any bulk lubricant film. However, at the same time the generated solvation pressures increase the load carrying capacity. Conversely, long chain molecules tend to inhibit solvation, thus show a decrease in the load carrying capacity, whilst through their wetting action reduce friction. Consequently, real lubricants should comprise molecular species which promote desired contact characteristics, as indeed is the case for most base lubricants with surmised properties of certain additives. The methodology presented underpins the rather empirical implied action of surface adhered films. This is an initial approach which must be expanded to fluids with more complex mix of species. If applicable, this could also be an alternative (potentially time saving) approach to Monte-Carlo simulations for molecular dynamics.
\end{abstract}

Keyword: Molecular level tribology, solvation, viscous shear, cavitation, nano-scale non-Newtonian friction 


\section{Introduction}

There is a progressive demand for accurate models to predict boundary friction between real (rough) surfaces. The main goal is to reduce frictional losses, and therefore, improve mechanical efficiency. Most frictional losses are as the result of any direct interaction of rough surface asperities. Nevertheless, a significant part of friction is also due to the shear of a thin lubricant film, usually trapped between opposing asperities of the contacting surfaces. In some applications an ultra-thin surface film is only possible, where molecular behaviour of liquid lubricant near surface asperities is of significance. These are usually lightly loaded contacts. However, in the micro-scale conjunctions of light to moderate load intensity a thin lubricant film adsorbed or entrained in between asperities can also behave in the same manner. This is also true of any lubricated conjunction at start-up or during inlet boundary reversal, where a coherent lubricant film cannot be assured.

New manufacturing techniques can now provide better control of surface topography (e.g. roughness, texturing). Molecular rheology of lubricant can also be improved by choosing an appropriate composition. This means that additives, having desired molecular interactions with solid bounding surfaces, are added to the base lubricants. It is hoped that the bulk rheology of the mixture would improve the load carrying capacity of the overall conjunction and possess low shear resistance. At the same time molecular behaviour near the smooth solid boundaries or around surface asperities would reduce friction when a coherent film is not formed. Most tribological conjunctions are subject to transient conditions, such as in start-up, inlet boundary reversals or because of application of cyclic loads. Therefore, interactions occur between the various phenomena at different physical scales; between bulk micro-scale lubrication and nano-scale localised interactions (e.g. around asperity tip pair contacts).

At micro-scale, viscous shear is the dominant mechanism underlying friction, as in hydrodynamic and elastohydrodynamic conditions. These mechanisms are better understood. At nano-scale, boundary interactions are affected by other kinetic laws such as intermolecular and surface energy effects. There is, of course, the critical interaction between these phenomena, particularly in the intervening scale. This interaction is between surface topography and lubricant rheology at short range (thin surface films of dimensions of several molecular diameters of the base lubricant or its additives). There is no deterministic model representing the formation of such ultra-thin films, particularly with respect to their interactions with the bulk tribological conditions. This study is an initial attempt to address this shortcoming.

At one end of the interaction range one can consider a single hemisphere. This represents a surface feature (such as an introduced dimple) or an idealised asperity tip, sliding against a perfectly smooth substrate, furnished with a uniform film of a

basic lubricant (of a single molecular composition). Such a lubricant may represent a thin protective layer or a sealant, which when subjected to shear forms a thin surface adsorbed film. Octamethycyclotetrasiloxane (OMCTS) is used as a low friction 
protective layer or a sealant in some applications, for example in siliconised rubber seals. These are often used in inhalation devices. OMCTS is a non-polar fluid with molecular diameter of around $1 \mathrm{~nm}$. Al-Samieh and Rahnejat [1] have shown that near molecularly smooth surfaces the behaviour of such a fluid is dominated by the solvation effect for films of up to several molecular diameters deep. Chan and Horn [2] have shown that there is discretised drainage of OMCTS from such conjunctions, indicating its near surface solvation effect. It is postulated that non-polar small molecular diameter spherical molecules (such as those of OMCTS) reduce localised viscous friction near asperity tips at nano-scale, which one may assume to be ideally smooth at this range. This is because solvation leads to dewetting of contacting surfaces.

At the other limit of this investigation, there are tribological conjunctions in microscale, where the load carrying capacity of the contact must be assured. Therefore, dewetting of the contiguous surfaces is not considered as a viable option. Consequently, long chain molecules are used to discourage density perturbation of the lubricant near the bounding contacting solid surfaces. Israelachvili [3] suggests that long chain molecules are best suited for this purpose and indeed are used regularly as additives to base lubricants as boundary friction modifiers. This is another postulate which should be investigated. In the current study the same sliding contact model is used, but with an idealised lubricant film, based on long chain molecules of Hexadecane or Tetradecane. These are used in diesel fuel, where they act as the lubricant in off-road vehicle fuel pumps. They intervene between the sliding teeth surfaces of pump gears.

The mechanism of delivery of the lubricant into the contact in both the examples investigated is assumed to be through hydrodynamics. The method of solution, therefore, combines hydrodynamics of the conjunction with solvation of the lubricant near the smooth surface of an assumed hemispherical feature. The effects of starvation and cavitation beyond the contact exit are also taken into account. Starvation affects the supply of lubricant into the contact, thus the thickness of film which influences

solvation. Inclusion of cavitation and lubricant reformation influences the load carrying capacity of the contact, which determines the instantaneous equilibrium load shares of viscous and surface force contributions. Hitherto, such a study has not been reported in open literature.

\section{Mathematical Model}

Figure 1 is a schematic representation of contact cross-section between a rigid hemispherical feature, sliding relative to a smooth flat semi-infinite elastic half space. Under the usual conditions encountered in lubricated contacts, a number of distinct regions would occur in the conjunction. There is the inlet wedge region, where the lubricant is entrained into the contact (in the opposite direction to the sliding motion), from a curved meniscus. This is followed by a region of parallel film (corresponding to high pressures) with any small strain localised deformation in accord with Hertzian assumptions (elastohydrodynamic region). At the exit region from the Hertzian zone, 
there is film rupture boundary, beyond which falling pressures below that of lubricant vaporisation leads to streamers; formation of vapour fingers in the lubricant flow. This is the cavitation region. Somewhere downstream of this region, lubricant film is assumed to reform. This rather orderly and now well understood lubricant flow characteristics is based on the principle of continuity of flow, which is fundamental to the bulk lubricant behaviour. Abd Al-Samieh and Rahnejat [4] showed that any near surface effect plays an insignificant role in continuity of flow, until the film thickness is gradually reduced to several molecular diameters of the lubricant. Then, discrete lubricant molecular behaviour pursues, followed by a stepwise drainage from the contact, roughly in accord with the molecular diameter of lubricant, which are assumed to be spherical. This finding conformed to the experimental observations of Chan and Horn [2] and predictions of Matsuoka and Kato [5].

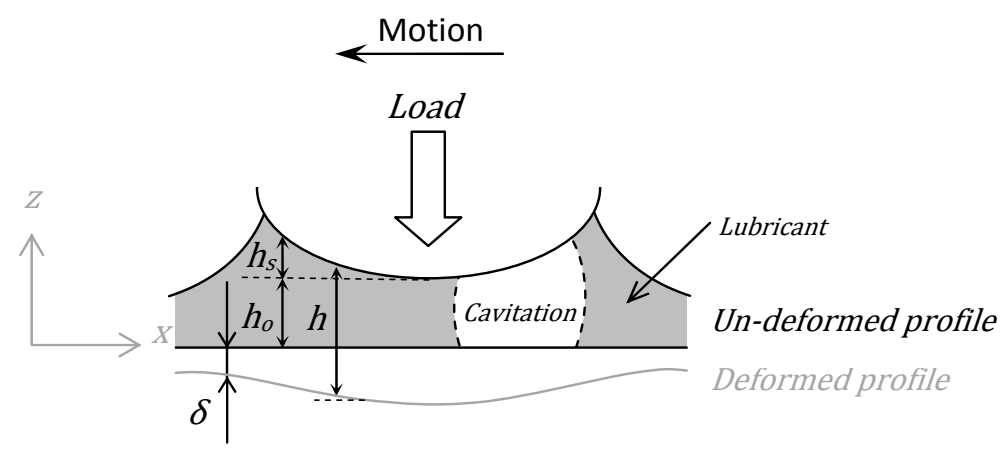

Figure 1. Lubricated conjunction

Therefore, for fairly thin films, the pressures generated in the conjunction are due to a number of mechanisms, whose individual contributions vary according to the gap size, $h(x, y)$ :

$$
h(x, y)=h_{0}+h_{s}(x, y)+\delta(x, y)
$$

where $h_{s}(x, y)$ is the local gap at any location within the conjunction and $h_{0}$ is the minimum clearance.

For inert surfaces and non-polar lubricants, assuming no direct contact between the surfaces, the total conjunctional pressure is due to hydrodynamics, van der Waals pressure and solvation:

$$
p_{T}=p_{h}+p_{w}+p_{s}
$$

where: $p_{h}$ is the hydrodynamic component of the pressure, $p_{w}$ is the van der Waals component and $p_{s}$ is the solvation contribution.

It should be noted that the contribution due to van der Waals interaction can become significant at almost vanishing separations. 
The load carrying capacity of the contact is obtained as:

$$
W=\int p_{T} d A
$$

where $p_{T}>0$ means that negative (attractive) pressures do not contribute to the load carrying capacity.

\subsection{Hydrodynamic Pressure}

Al-Samieh and Rahnejat [1] and Matsuoka and Kato [5] solved the Reynolds equation to obtain the hydrodynamic contribution. For the point contact geometry, the two dimensional Reynolds equation is:

$$
\frac{\partial}{\partial x}\left[\frac{\rho h^{3}}{\eta} \cdot \frac{\partial p_{h}}{\partial x}\right]+\frac{\partial}{\partial y}\left[\frac{\rho h^{3}}{\eta} \cdot \frac{\partial p_{h}}{\partial y}\right]=12\left\{U \frac{\partial \rho h}{\partial x}+V \frac{\partial \rho h}{\partial y}+\frac{d}{d t}(\rho h)\right\}
$$

where, $U$ is the speed of entraining motion of the lubricant into the conjunction, being the average speed of the contacting surfaces (or half the speed of sliding in the case studied here) and $V$ is the speed of any side-leakage of lubricant out of the contact area.

The hydrodynamic load carrying capacity of the contact is confined to the regions of full film, prior to a region of cavitation beyond a film rupture point. Swift-Stieber exit boundary conditions is used: $\bar{P}=\partial \bar{P} /\left.\partial x\right|_{\text {cavitation }}=0[6,7]$. Cavitation, though, plays a very significant role in the disruption of free surface film uniformity as desired for thin protective surface films. Additionally, Reynolds equation is not suited to the prediction of the cavitation region, where the interface between the lubricant and air causes loss of laminar flow. This is an underlying assumption for Reynolds equation.

To overcome this shortcoming the cavitation model proposed by Elrod [8] is used here. This model modifies the Reynolds equation to take into account the boundary between a full film region and that representing a cavitation region. The approach approximates the fully demarcated vaporization cavitation boundary condition proposed by Jakobsson and Floberg [9] and Olsson [10] (now referred to as the JFO boundary condition). In the simplified, but computationally more efficient Elrod method a fraction film content ratio, $0<\theta \leq 1$ is defined. The hydrodynamic pressure can now be defined as:

$$
p_{h}=g \beta \ln \theta+p_{c}
$$

where cavitation is assumed to occur at the constant pressure, $p_{c}$ under the current isothermal analysis, $\beta$ is the lubricant bulk modulus and $g$ is a switching function:

$$
g= \begin{cases}1 \Rightarrow & \text { Full film, if } \theta \geq 1 \\ 0 \Rightarrow & \text { Cavitation, if } 0<\theta<1\end{cases}
$$

Now for the full film region, Reynolds equation is modified to: 


$$
\begin{aligned}
\frac{\partial}{\partial x}\left[\frac{\rho_{c} h^{3}}{\eta} g \beta \frac{\partial \theta}{\partial x}\right] & +\frac{\partial}{\partial y}\left[\frac{\rho_{c} h^{3}}{\eta} g \beta \frac{\partial \theta}{\partial y}\right] \\
& =12\left\{U \frac{\partial \theta \rho_{c} h}{\partial x}+V \frac{\partial \theta \rho_{c} h}{\partial y}+\frac{d}{d t}\left(\theta \rho_{c} h\right)\right\}
\end{aligned}
$$

Referring to (6), it is clear that in the cavitation region the Couette flow only operates, thus:

$$
\left\{u \frac{\partial}{\partial x}+v \frac{\partial}{\partial y}+\frac{\partial}{\partial t}\right\}\left(\theta \rho_{c} h\right)=0
$$

It is reasonable to assume negligible side leakage from the contact with thin films and at relatively low sliding speeds, thus: $V=0$. With this assumption taken into account, it is clear that the cavitated region is governed by a balance between the squeeze film effect (the ultimate term in the last equation) and the drainage of the fluid from the contact on the account of hydrodynamics alone. Separation of the surfaces on account of squeeze; $\partial \theta h / \partial t>0$ increases the chance of cavitation, which is the same as an increase in the speed of entraining motion (Al-Samieh and Rahnejat [11]). One should then expect reduced cavitation at lower sliding speeds as an outcome of the analysis, which would encourage better lubricant distribution on the free surface post film reformation. This is a desired outcome in any reciprocating contact.

Lubricant rheology, therefore, is critical to the maintenance of a continuum. Yet, density alters as a direct result of the different kinetic laws that co-exist in the nanoscale. In bulk it alters with hydrodynamic pressure, and near the solid barrier it has a higher density profile due to the constraining effect of solvation pressure (section 2.2). The bulk density variation with the contact pressure for mineral oils is given by Dowson and Higginson [12]:

$$
\rho=\rho_{o}\left(1+\frac{0.6 \times 10^{-9} \times p_{h}}{1+1.7 \times 10^{-9} \times p_{h}}\right)
$$

As a first approximation this is taken to also represent the density-pressure dependency in this analysis. Matsuoka and Kato [5], Abd Al-Samieh and Rahnejat [4] and Al-Samieh and Rahnejat [1, 11] also assumed the same for the bulk hydrodynamic behaviour of the lubricants such as those described above and used in the current analysis. Clearly, for the cavitated region: $\rho=\rho_{c}$ when $p=p_{c}$.

Lubricant viscosity-pressure variation can be predicted using the Roelands' equation [13]:

$$
\eta=\eta_{o} e^{\alpha p_{h}}
$$

where $\alpha=\frac{1}{p_{h}}\left(\ln \eta_{o}+9.67\right)\left\{\left[1+p_{h} /\left(1.98 \times 10^{8}\right)\right]^{Z}-1\right\} / p_{h}$ and $Z=\alpha_{o} /\left[5.1 \times 10^{-9}\left(\ln \eta_{o}+\right.\right.$ 9.67)]. 


\subsection{Solvation Pressure}

For very smooth surfaces, the solvation effect dominates in gaps of several molecular diameters of the intervening fluid. The phenomenon is as the result of density variation of liquids near solid boundaries [3]. The effect is significant in nano-scale conjunctions, inducing a structureless conjunctional environment which disrupts the comfort of a hydrodynamic continuum and thus proper wetting of the contiguous solids. Paradoxically, the dewetting action of solvation can guard against the formation of a meniscus, which otherwise would contribute to the work of adhesion.

It is postulated that the effect of solvation is more pronounced for small fluid molecules such as those of perfluoropolyether and Octamethylcyclotetrasiloxane, which have nominally spherical molecules of $1-1.5 \mathrm{~nm}$ diameter. This effect is almost negligible for long chain molecules [3]. The contact density variation near the smooth solid surfaces takes the form of (Henderson and Lozada-Cassou [14] and Evans and Perry [15]) :

$$
\rho=K T[\rho(h)-\rho(h \rightarrow \infty)]
$$

where $\rho(h \rightarrow \infty)$ is the corresponding value for a single surface, implying that $h>>a$. Therefore, it tends to the bulk density of the fluid, whose variation may be assumed to follow equation (9).

The density variation near the constraining barriers gives rise to a monotonic exponential pressure variation, which is alternately attractive-repulsive. This variation is explained in some detail by Israelachvili [3], Teodorescu et al [16] and, Gohar and Rahnejat [17], resulting in the discrete fluid drainage from the contact conjunction. Israelachvili [3] proposed a solvation pressure of the following form:

$$
p_{s}=-C e^{-h / a} \cos \left(2 \pi \frac{h}{a}\right)
$$

where: $C=K T \rho(h \rightarrow \infty)$, which for the cases considered here: for OMCTS $C=172 M P a$, Hexadecane $C=62 M P a$ and Tetradecane $C=49 M P a$ (Chan and Horn [2]). Note that the value of $C$ is reduced as the size of the molecule increases. Size is not represented by molecular diameter for long chain molecules, which are rather similar to strings. In fact the diameter of Hexadecane is only $0.4 \mathrm{~nm}$.

It is clear that the solvation pressure is only significant for small values of $h / a$.

\subsection{Van der Waals Pressure}

In nano-scale conjunctions other forms of kinetic interaction may also contribute, depending on the molecular structure of the fluid and the atomic structure of the bounding solids. If the fluid is considered as non-polar and the surfaces as inert, then long range van der Waals interactions between the fluid molecules and atoms of the surfaces and between the surfaces themselves play a role. These are weak attractive forces in the nano-scale, but can become very significant at closer range. 
The van der Waals forces arising from these interactions can be obtained using Lifshitz theory [18]. For nano-scale conjunctions, the procedure is detailed by Abd AlSamieh and Rahnejat [4]. The current study addresses the nano-scale range, where some marginal contributions due to van der Waals interactions may be expected. Depending on the contact geometry, Israelachvili [3] provides a simple expression. For flat surfaces (which may be assumed here because of the localized elastic deformation in the central small contact area in figure 1):

$$
p_{w}=-\frac{A_{h}}{6 \pi h^{3}}
$$

where $A_{h}$ is the Hamaker constant, its value depends on the atomic structure of contacting bounding solids and the molecular composition of the intervening fluid. Its value is usually in the range: $10^{-21}-10^{-19} \mathrm{~J}$, in this case: $A_{h}=1.4 \times 10^{-20} \mathrm{~J}$.

\subsection{Contact Deflection}

Contact deflection, required in equation (1) can now be calculated, using the elasticity potential equation (Johnson [19] and Gohar and Rahnejat [17]):

$$
\delta(x, y)=\frac{1}{\pi E^{\prime}} \iint \frac{p_{T}\left(x^{\prime}, y^{\prime}\right)}{\sqrt{\left(x-x^{\prime}\right)^{2}+\left(y-y^{\prime}\right)^{2}}} d x^{\prime} d y^{\prime}
$$

where the deflection at a point $(x, y)$ is determined due to all the generated pressures at points $\left(x^{\prime}, y^{\prime}\right)$. When a computational grid is made with the pressure distribution, $p_{k, l}$, this equation can be stated as:

$$
\delta_{i, j}=\sum_{k} \sum_{l} D_{i, j}^{k, l} p_{k, l}
$$

where $D_{i, j}^{k, l}$ are the influence coefficients (Johnson [19]). The reduced elastic modulus is

$$
E^{\prime}=\left(\frac{1-\nu_{1}^{2}}{E_{1}}+\frac{1-\nu_{2}^{2}}{E_{2}}\right)^{-1}
$$

\section{Method of Solution}

A solution can now be obtained for $\theta, h, p, \delta, \rho$ and $\eta$, by representing equation (7) in finite differences and obtaining a solution through low relaxation effective influence Newton-Raphson method with Gauss-Seidel iterations (Al-Samieh and Rahnejat [1], Teodorescu et al [16] and Chong et al [20]). The procedure places the hemispherical feature at consecutive reducing separations $h_{0}$ from the surface of the flat semi-infinite plane. With an assumed speed of entraining motion $(U)$ and time step, this corresponds 
to the imposition of a rate of rigid body squeeze $(\partial h / \partial t)$. Therefore, the conditions at each quasi-static step at various separations $h_{s}(x, y)$ may be regarded as rheonomic nonholonomic constraints. The simulation results in section 5 explore contact conditions with different rates of approach. The convergence criterion used in each quasi-static step is:

$$
\frac{\sum_{i} \sum_{j}\left|\theta_{i, j}^{n}-\theta_{i, j}^{n-1}\right|}{\sum_{i} \sum_{j} \theta_{i, j}^{n}} \leq \epsilon_{\theta}
$$

where $n$ is the iteration counter. If the above criterion is not satisfied, then:

$$
(g \theta)_{i, j}^{n}=(g \theta)_{i, j}^{n-1}+\omega \Delta \theta_{i, j}^{n}
$$

The process is then repeated at the same separation. When the convergence criterion is met, the separation $h_{0}$ is reduced by a molecular diameter of the fluid and the entire iterative procedure is repeated again. In this manner the conjunctional behaviour is noted for different quasi-static steps at progressively reduced separations.

\section{Conjunctional Friction}

Friction generated by the ultra-thin adsorbed films on nominal molecularly smooth surfaces follow non-Newtonian shear due to chemical reactions which are based on thermal activation. Eyring [21] developed a model which described the viscosity of fluids under such circumstances. Eyring's model assumes that the motion of a volume of fluid molecules takes place in the presence of a cage-like potential barrier by their mere closely-packed arrangement in the bulk. In order to overcome this potential and escape the cage, the fluid molecules have to surmount the activation barrier potential. This is the thermal activation model which Eyring modified to include the effects of prevailing pressure and shear (Figure 2) as:

$$
E_{y}=Q-p \Omega-\tau \phi
$$

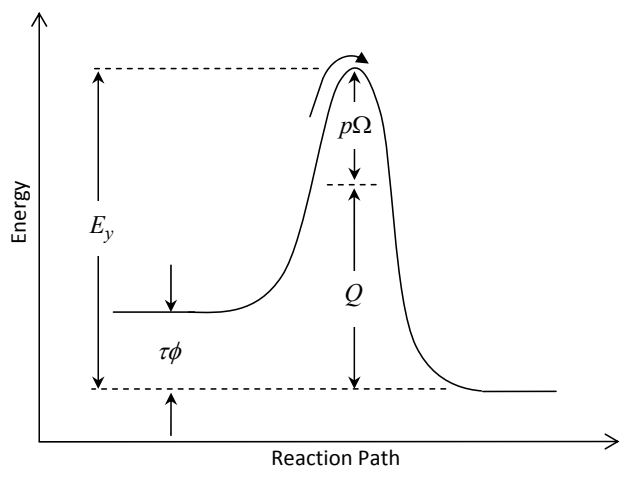

Figure 2. The potential barrier in thermal activation 
The shear stress acts on a stressed activation volume, $\phi$, which may be conceived as a process coherence volume. This can be interpreted as the size of a moving segment in unit shear, whether it would be a molecule or a dislocation line. Therefore, during sliding motion the potential barrier is repeatedly overcome. The average barrier-hopping time for a single molecule is predicted using the Boltzmann distribution with a regular series of barriers and a high stress limit (i.e. $\tau \phi / K T>1$ ). According to Briscoe and Evans [22], the shear stress is a function of velocity, pressure and temperature. For constant velocity and under isothermal conditions, it can be expressed as:

$$
\tau=\tau_{0}+\xi p
$$

where : $\xi=\frac{\Omega}{\phi}$

The Eyring shear stress is determined as : $\tau_{0}=\frac{1}{\phi}\left[K T \ln \left(\frac{U}{v_{0}}\right)+Q\right]$. where $v_{0}$ is the characteristic velocity related to the frequency of the process.

The sliding velocity, however, can change according to the operating speed of the contact. To account for this, the value of $\tau_{0}$ is adjusted at a constant pressure, $p_{v}$ as:

$$
\tau_{0}=\frac{K T}{\phi} \ln U+\tau_{1}-\xi p_{v}
$$

where $\tau_{1}=\frac{1}{\phi}\left[Q-p_{v} \Omega+K T \ln v_{0}\right]$, and for the fluids used in this analysis: $K T / \phi \approx$ $3.4 / A, \tau_{1} \approx 11.3 / A, p_{v} \approx 100 / A$ all in $n N / m^{2}$ and $\xi \approx 0.16$ [23]. Note, the contact

area is taken as the area of the computational grid: $A=\iint d x d y$ and thus friction is obtained as:

$$
F=\iint \tau d x d y ; \tau>0
$$

\section{Results and Discussion}

Series of simulations have shown that while the conjunction is separated by a molecularly thin film, the combined effects of solvation and hydrodynamics dominates, while the long range van der Waals interactions play an almost insignificant role.

When the gap is greater than several molecular diameters of the intervening fluid, hydrodynamics dominate. As the gap is reduced the effect of solvation becomes increasingly important. For the fluid used (OMCTS) in the first part of this analysis, with molecular diameter $a \approx 1 \mathrm{~nm}$, an incipient solvation effect begins around gaps of the order of $8 \mathrm{~nm}$ (figure 3). Nevertheless, hydrodynamics always plays the key role of entraining a thin film of lubricant into the conjunction through inlet wedge effect. 


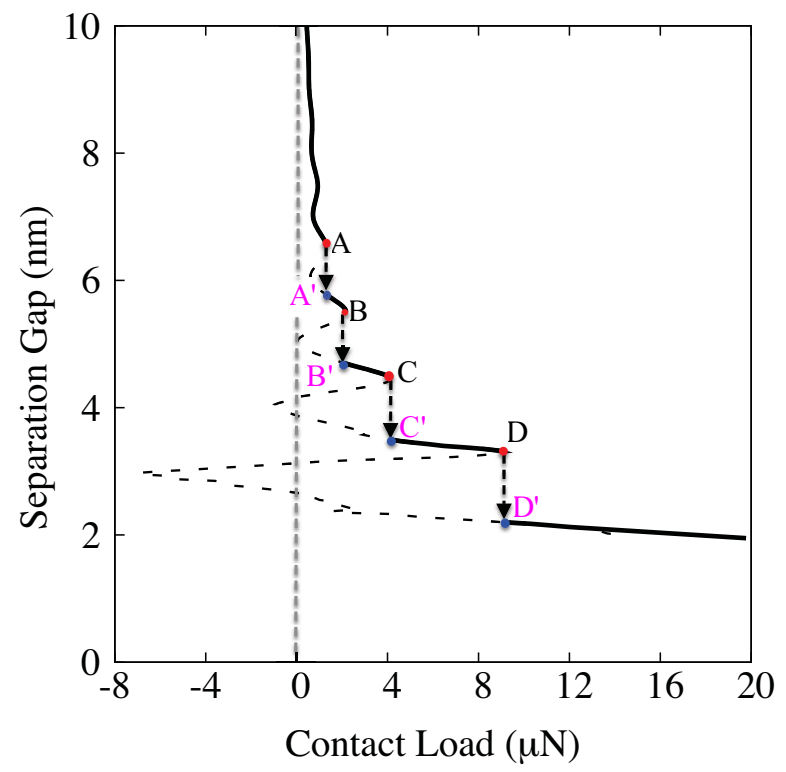

Figure 3. Discretized contact characteristics in ultra-thin smooth conjunctions

Figure 4(a) shows an isobaric plot of conjunctional pressures. This comprises 4 regions of interest: (1) the inlet meniscus/wedge, (2) the contact proper, (3) a cavitation region and (4) reformation zone (as postulated in description of figure 1). Each region plays a key role. The inlet meniscus acts as the reservoir supplying the contact with a film of lubricant. Lubricant ahead of the contact provides the meniscus liquid bridge through wetting action of the lubricant on the contiguous surfaces, and is indicated by the inward curved isobar ahead of the contact. Under these circumstances, for simplicity it is assumed that a meniscus is formed at a pressure close to that of lubricant's vaporization pressure $p_{c}$. With entraining motion of the lubricant through the inlet wedge the generated pressures rise. The contact proper comprises a series of almost concentric isobars with increasing pressures towards the rear lubricant exit constriction from the contact. This is a characteristic of hydrodynamic pressures, except that solvation effect in such diminutive gaps causes pressure fluctuations superimposed upon the hydrodynamic pressure profile. Figure 4(b) is a cut through the centre line of the isobaric plot, which shows these pressure fluctuations.

The region of high pressures is immediately followed by oil film rupture, not unlike the behaviour of any hydrodynamic conjunction. With lubricant pressures falling below its vaporization pressure beyond the lubricant rupture point, cavitation occurs. The depth of cavitation is governed by the speed of entraining motion in the contact, as well as the gap size. The former is contributed by hydrodynamics, whilst the latter determines the extent of solvation. The fluid in the cavitation region is a mix of lubricant and vapour. Beyond this, in the trailing edge of the contact, the pressures are assumed to return to the atmospheric value. This implies the existence of a film reformation region, where the vapour escapes through the liquid. Figure 4(b) shows the trend towards reformation. The larger the extent of cavitation and reformation regions become, the 
greater is the deviation from uniformity of lubricant height upon the free surface of the semi-infinite plane after the exit constriction. It is natural to assume that clumps of lubricant may be formed on any surface beyond the film rupture region if many such hemispherical features or other textures slide along the flat substrate. The analysis here shows that even with assumed steady state condition the transient nature of solvation and its interplay with hydrodynamics can result in non-uniform distribution of lubricant on surfaces.

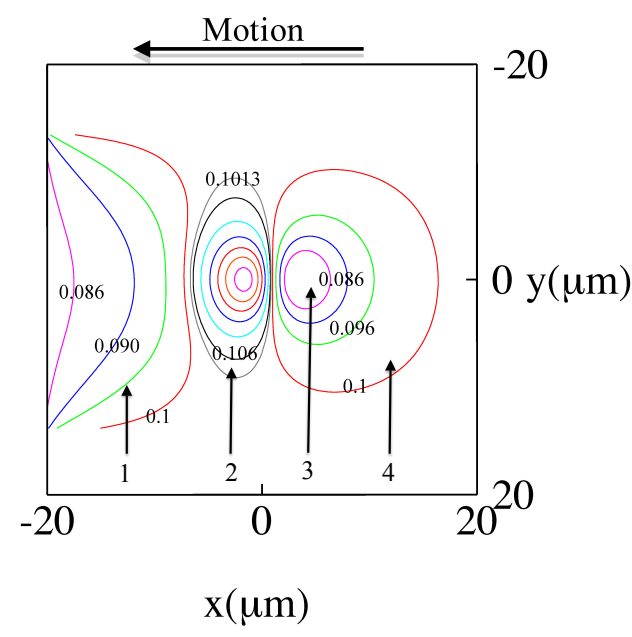

(a) Isobaric pressure plot (MPa)

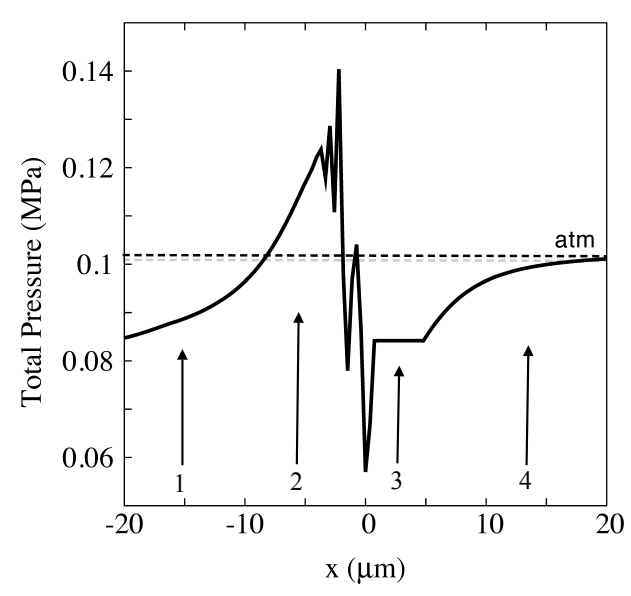

(b) Centre-line conjunctional pressures

Figure 4. Pressure variation in an ultra-thin gap of $8 \mathrm{~nm}$

Now returning to figure 3, as the gap is reduced, fluid film discretization occurs (as noted by Matsuoka and Kato [5], and Al-Samieh and Rahnejat [1]). Layers of lubricant molecules are drained in a step-wise fashion from the conjunction, in this case at gap intervals of $1 \mathrm{~nm}$; the diameter of spherical molecules of OMCTS. In each step a further increase in load or a corresponding squeeze action is needed before another layer of lubricant is ejected out of the contact. This means that at a given sliding speed, whilst the hydrodynamic inlet flow follows Newtonian slow viscous action, the drainage from the contact does not conform to the continuity of flow condition. Solvation in effect accounts increasingly for load carrying capacity of the contact. It disrupts the structured nature of fluid flow and essentially promotes its dewetting. There is, of course, a limit to this height, determined by the meniscus pressure at the inlet (Abd Al-Samieh and Rahnejat [4]). The dotted lines in figure 3 indicate the oscillatory behaviour of solvation, in alternate attractive-repulsive action. The loss of load carrying capacity, indicated by the dotted line is purely theoretical as the hydrodynamic pressure at the nib of the contact guards against this effect, unless no entraining motion is to take place (such as in cessation of sliding).

Figure 5 shows a series of pressure distributions with the corresponding film shapes and fraction film ratios for steadily decreasing gaps in line with the steps indicated in figure 3. As the gap is reduced (e.g. from A to $A^{\prime}$ ) at the same sliding speed, 
the contribution due to solvation pressure is increased. This reorders the molecular content of the conjunction within the contact proper, followed by the drainage of a row of molecules. The film shapes show this discrete molecular drainage. In the intervening intervals (e.g. A' to B) an increase in the contact load (i.e. integrated pressure distribution) is due to molecular disposition to regiment rows of molecules within the conjunction. With increased pressures and thin films the depth of cavitation is increased directly as the result of the dewetting action of solvation. With larger cavitation and assuming a fairly uniform surface topography, a longer time, hence sliding distance, is required before film reformation is achieved through fluid surface tension. Therefore, under dynamic conditions with varying height of the surface features, it is clear that uneven distribution of film would result on the surfaces. The results indicate that this non-uniformity would be exacerbated at lower gap sizes. 

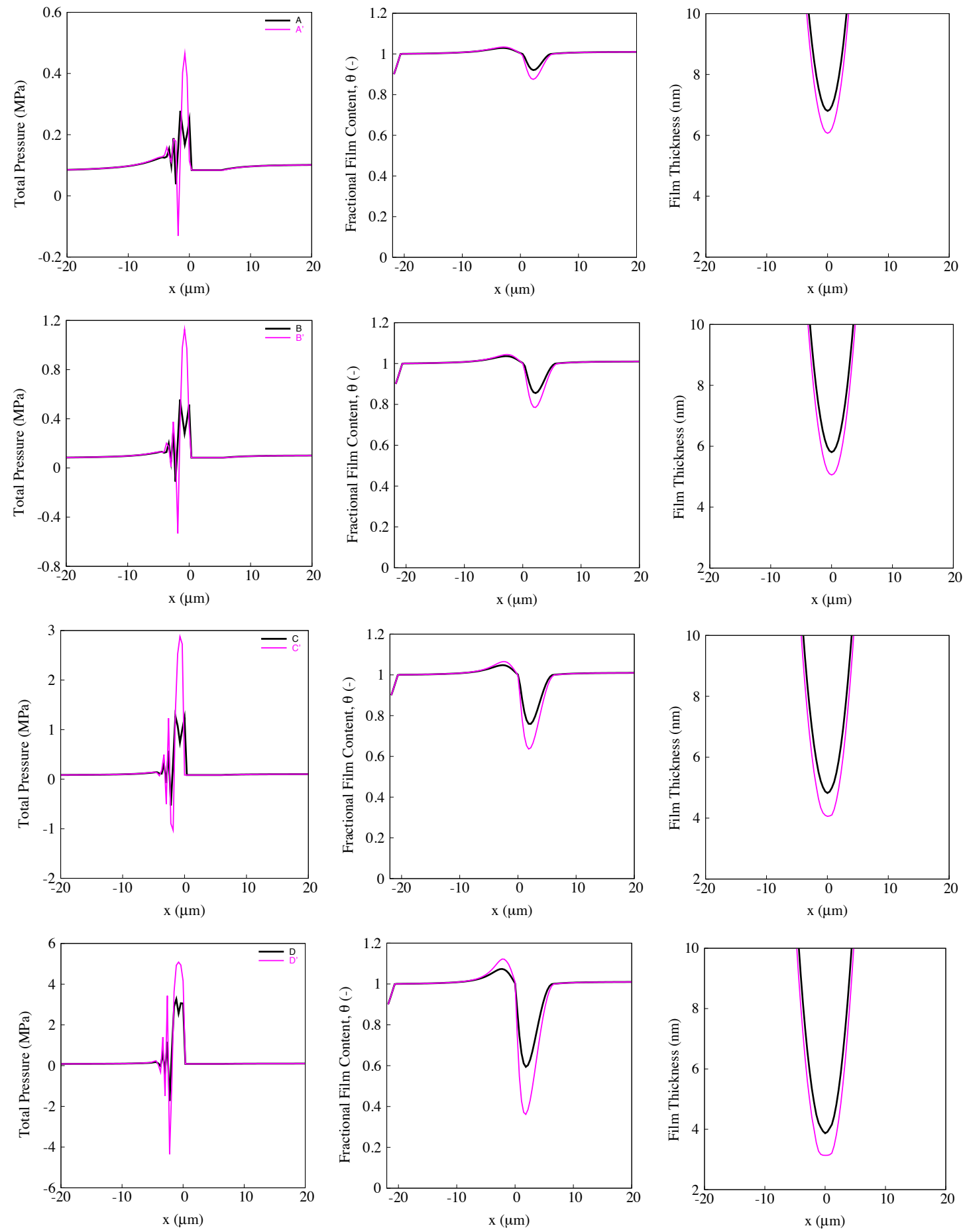

Figure 5. Conjunctional conditions at a steadily reduced flying height

Hydrodynamic entrainment of the lubricant also has its own better known influence upon film rupture and cavitation. The height of lubricant at the meniscus and its distance to the leading edge of contact is critical to its wetting action (Hamrock and Dowson [24], Wedeven et al [25]). These are affected by the sliding speed. A faster sliding speed requires a longer inlet distance (i.e. a larger meniscus) in order to avoid starvation of the contact. However, this can also lead to thicker contact films and greater 
depth of cavitation. These effects lead to conditions that are contrary to the purpose of maintaining a better distribution of liquid lubricant in many applications.

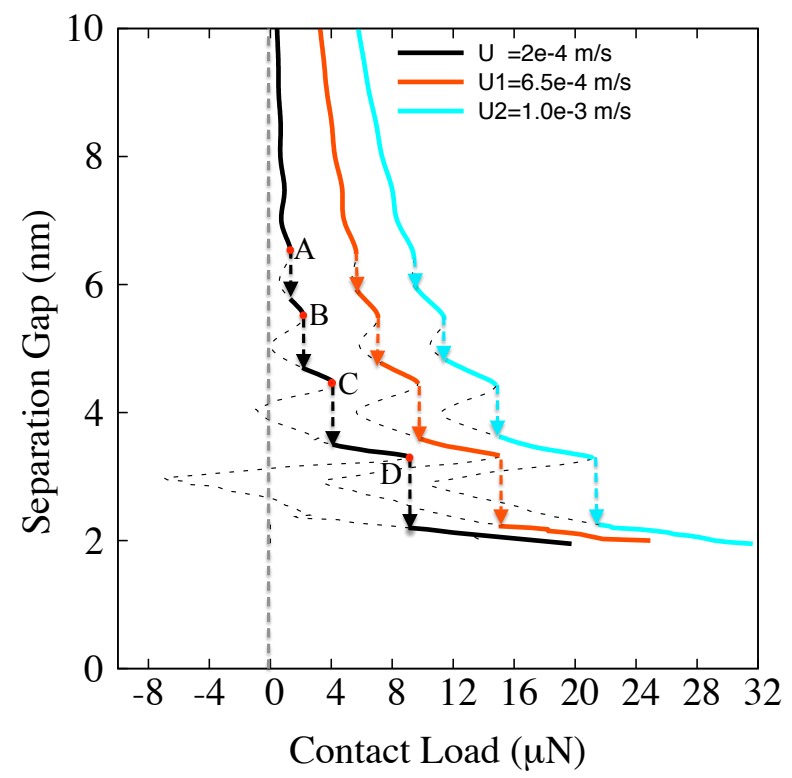

Figure 6. Conjunctional characteristics with increasing slider speed

Figure 6 shows that the discretisation effect is slightly delayed with increasing speed of entraining motion. This delayed effect is caused by a rise in film thickness, thus more pronounced hydrodynamic action. Figure 7 shows a series of pressure distributions with their fraction film ratios, corresponding to different speeds of entraining motion in figure 6. Note that the rise in pressures is due to hydrodynamics in the inlet nib to the contact (this diminishes with smaller gaps). The solvation contribution remains the same for all the speeds in the same gap size. Interestingly, the depth of cavitation is increased as normally would with enhanced contact pressures, directly as the result of increased speed at the same effective contact load. Hence, reduced gap and increased sliding speed cause non-uniformity in free surface oil film, the former due to solvation and the latter due to hydrodynamic action.

Another key factor is friction. Assuming very smooth surfaces, at the molecular level separations here, friction is mainly due to viscous action of a lubricant film or introduced by a layer of molecules in drainage in accord with the activation energy required to dislocate them. There is clearly insignificant friction in the cavitation region. 

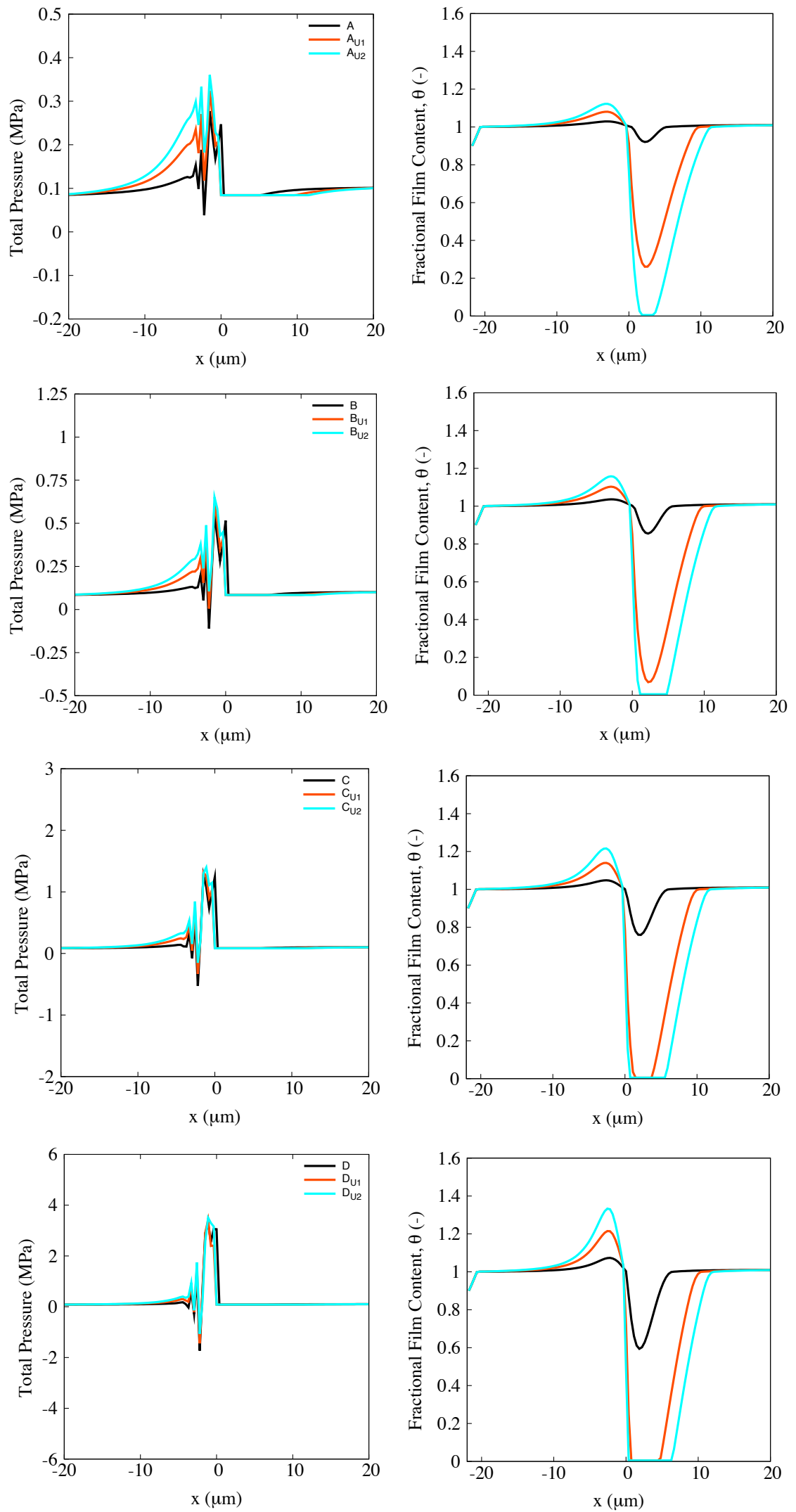

Figure 7. Liquid film discretization at increasing speed of entraining motion 
Figure 8 shows a plot of friction versus the conjunctional load (at a steadily reducing gap). The quasi-linear relationship between any subsequent instances of drainage is due to shear of a lubricant film, with a slope, which may be interpreted as a coefficient of friction. This alters slightly with both the sliding speed and contact pressures. The linear slope is as the result of changes in lubricant viscosity and film thickness, both affected by increasing load between steps of lubricant discretisation. The slope follows viscous friction. Seth et al [26] studied slip of microgel paste particles against a solid boundary in the presence of water molecules. They showed that at each step of separation the slip of microgel particles along the wall follow viscous shear.

Referring back to figure 8 , the change with pressure is almost insignificant at a given gap size as this is dominated by almost unchanged solvation pressure. However, during drainage a rise in friction occurs with no additional contact load. This is the effort expended to eject a row of molecules. In such instances interpretation of friction based on viscous action (or an effective coefficient of friction) is inappropriate. Here one may regard friction as breaking of cohesive intermolecular bonds during discrete drainage from the contact region or a volume of fluid (i.e. a row of molecules) overcoming an activation barrier to drain from the contact. With increased sliding speed thicker films cause delays in the same characteristics.

Friction characteristics of thin films are quite complex, because they are affected by their actions near solid barriers. This is not only true for OMCTS studied so far, but also for low shear strength films formed by all additives in micro-scale conjunctions in a large variety of engineering applications. One case already mentioned is the action of additive molecules such as Hexadecane in diesel, where the latter also acts as a lubricant, for example in off-road vehicles' fuel pumps. In these cases the surface effects are likely to be concentrated around smooth tips of asperities, which are larger in dimension than the molecular size of the surface active additives. Figure 9(a) shows a similar friction characteristic for Hexadecane, if it were to be used instead of OMCTS in the current study under otherwise exact simulation conditions. However, note that in the case of Hexadecane a small rise in friction is only discerned during lubricant drainage. This is because Hexadecane has long and narrow molecular structure which inhibits solvation (figure 9(b)). Thus, the discrete nature of drainage is reduced. This improves friction, at the expense of better wetting of contact. Hence, a quest to reduce wetness of the contact can have significant drawbacks in terms of friction. However, in certain cases this is a desired function, such as in seals of inhalation devices containing volatile mixtures.

The current analysis is isothermal, thus the effect of friction is understated. It is clear that the heat generated as the result of friction would deplete the film thickness, the load carrying capacity of the contact and exacerbate the effect of cavitation. 


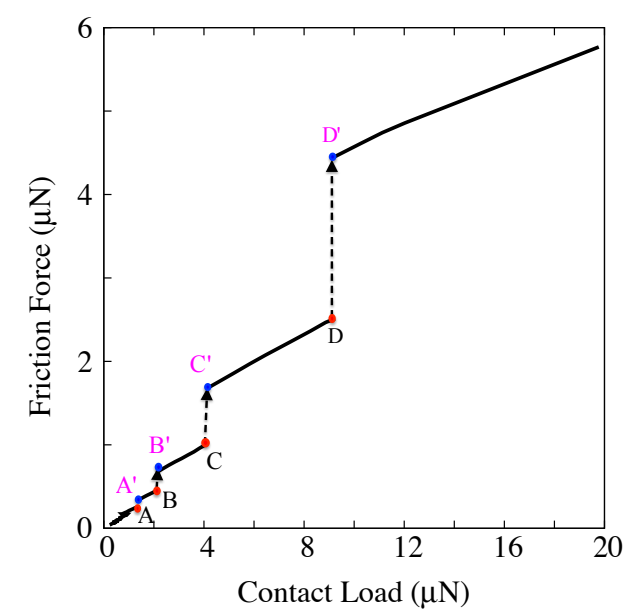

(a) Load-friction characteristics

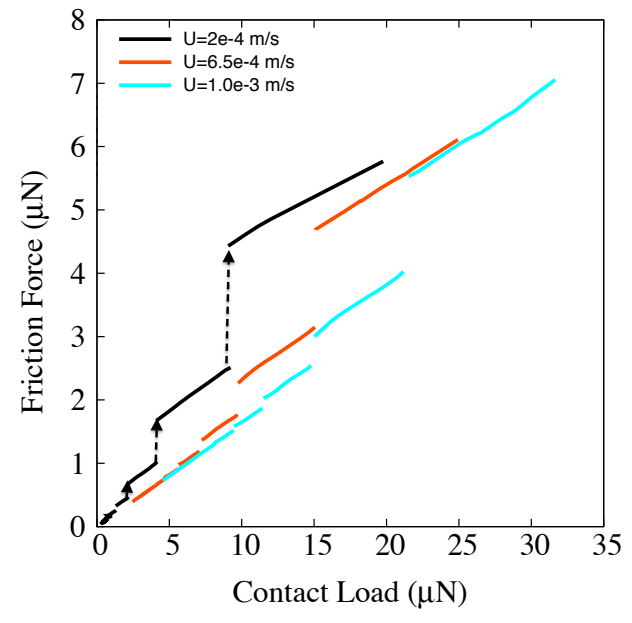

(b) Effect of increased sliding velocity

Figure 8. Friction characteristics of OMCTS

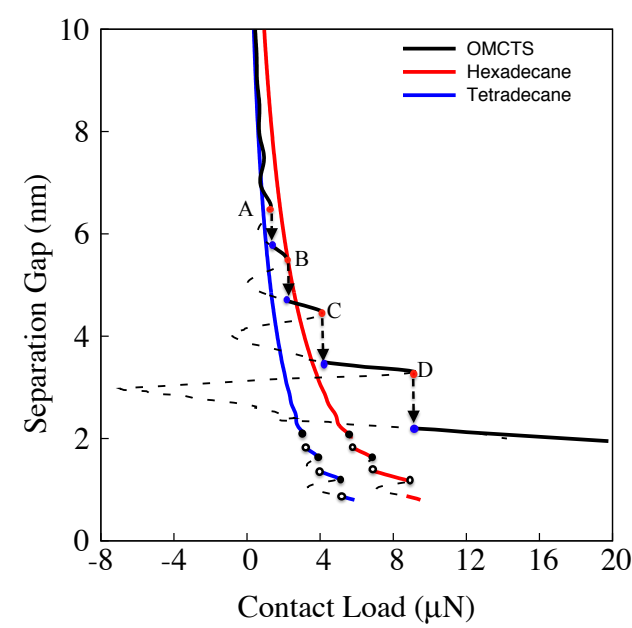

(a) Contact load vs. Separation Gap

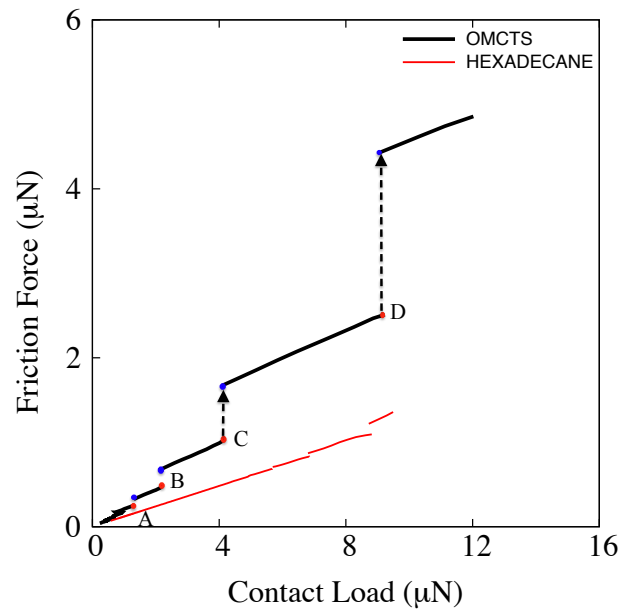

(b) Friction force vs. Contact Load

Figure 9. Friction characteristics of different species of molecules

The analysis shows that for some conjunctions one paradoxically needs the dewetting action of solvation, but uniformity, which is afforded by hydrodynamics. Hence, control of molecular composition of lubricants may be fundamental to future research in ultra-thin film devices.

\section{Conclusion}

There is a strong correlation between lubricant molecular structure and tribological behaviour of a conjunction. This becomes even more obvious when the conjunction is very small, at asperity level. The current paper investigates the interaction between local asperity or small feature deflection and molecular-level lubricant drainage as well as 
their impact on friction and local load carrying capacity of the lubricant. It is found that while long chain molecules inhibit discrete drainage of the lubricant from the contact leading to lower friction, they also lower asperity level load carrying capacity (see figure $9(\mathrm{a}))$ because of the absence of any significant solvation pressure. This can potentially lead to increased metal-to-metal interactions. Therefore, an optimal additive blend must consist of a variety of molecular species. The optimal proportion in the blend, should consider surface topography (e.g. roughness, texturing) together with molecular composition of the lubricant. For real lubricants, comprising an assortment of molecular species, the current model needs to be extended to take into account molecular mix as the result of competition near the solid boundaries. This would be affected not only by solvation of the molecules near the boundary solids, but also by the interaction between the various molecules themselves. More work will be required in the future to take these issues into account.

\section{Acknowledgement}

The authors acknowledge the technical support from partners and sponsorship provided by the EPSRC through the ENCYCLOPAEDIC program grant.

\section{References}

[1] Al-Samieh, M. F. and Rahnejat, H., Ultra-thin lubricating films under transient conditions , Journal of Physics D: Applied Physics, 34: 2610-2621, (2001)

[2] Chan, D. Y. C. and Horn, R. G., The drainage of thin liquid films between solid surfaces, J. Chem. Phys., 83: 5311-24 (1985)

[3] Israelachvili, J. N., Intermolecular and surface forces, Academic press, London (1992)

[4] Abd Al-Samieh, M. F. and Rahnejat, H., Nano-lubricant film formation due to combined elastohydrodynamic and surface force action under isothermal conditions, Proc. IMechE Part C: J. Mech. Eng. Sci., 215: 1019-29, (2001)

[5] Matsuoka, H. and Kato, T., An ultra-thin liquid film lubrication theory calculation method of solvation pressure and its applications to EHL problem, Trans. ASME, J. Tribology, 119: 217$26(1997)$

[6] Swift, W., The stability of lubricating in journal bearings, Proc. of the Institute Civil Engineers, 233: 267-288 (1932)

[7] Stieber, W., Hydrodynamische Theorie des Gleitlagers, Das Schwimmlager, V.D.I Verlag, Berlin (1933)

[8] Elrod, H. G., A cavitation algorithm, Trans. ASME, J. Lubn. Tech., 103: 350-354 (1981)

[9] Jakobsson, B. L. and Floberg, L., The finite journal bearing considering vaporization, Transactions of Chalmers University of Technology, (1957)

[10] Olsson, K. O., Cavitation in dynamically loaded bearings, Transactions of Chalmers University of Technology, (1965)

[11] Al-Samieh M. F. and Rahnejat H., Physics of lubricated impact of a sphere on a plate in a narrow continuum to gaps of molecular dimensions, J. Phys. D: Appl. Phys., 35: 2311-2326, (2002)

[12] Dowson, D. and Higginson, G. R., Elasto-hydrodynamic lubrication: The fundamentals of roller and gear lubrication, Pergamon Press, Oxford (1966)

[13] Roelands, C. J. A., Correlational aspects of the viscosity-temperature-pressure relationships of lubricating oils, TU Delft, Delft University of Technology (1966) 
[14] Henderson D. and Lozada-Cassou, M., A simple theory for the force between spheres immersed in a fluid, Journal of Colloid and Interface Science, 114(1): 180-183 (1986)

[15] Evans, R. and Parry, A. O., Liquids at interfaces: What can a theorist contribute?, J. Phys., Part B:, Condensed Matter Phys., 2, SA15 (1990)

[16] Teodorescu, M., Balakrishnan, S. and Rahnejat, H., Physics of ultra-thin surface films on molecularly smooth surfaces, Proc. IMechE Part N: Journal of Nanoengineering and Nanosystems, 220(1): 7-19 (2006)

[17] Gohar, R. and Rahnejat, H., Fundamentals of tribology, Imperial College Press, London, 2008.

[18] Lifshitz, E. M., The theory of molecular attractive forces between solids, Soviet Phys. JETP (Engl. Transl.), 2: 73-83 (1956)

[19] Johnson, K. L., Contact Mechanics, Cambridge University Press, Cambridge, 1985.

[20] Chong, W.W.F., Teodorescu, M. and Vaughan, N.D., Cavitation induced starvation for pistonring/liner tribological conjunction, Tribology International, 44: 483-497 (2011)

[21] Eyring, H., Viscosity, plasticity, and diffusion as examples of absolute reaction rates, Journal of chemical physics, 4: 283-291 (1936)

[22] Briscoe, B. J. and Evans, D. C. B., The shear properties of langmuir-blodgett layers, Proceedings of the Royal Society of London.Series A, Mathematical and Physical Sciences, 380(1779): 389-407 (1982)

[23] He M., Blum A.S., Overney G. and Overney R.M., Effect of interfacial liquid structuring on the coherence length in nanolubrication, Physical Review Letters, 88(15): 154302(4) (2002)

[24] Hamrock, B. J. and Dowson, D., Elastohydrodynamic lubrication of elliptical contacts for materials of low elastic modulus: Part 1- fully flooded conjunction, Journal of Tribology, 100: 236-245 (1978)

[25] Wedeven, L. D., Evans, D. and Cameron, A., Optical analysis of a ball bearing, Journal of Tribology, 93: 349-363 (1971)

[26] Seth, J.R., Cloitre, M. and Bonnecaze, R.T., Influence of short range forces on wall-slip in microgel pastes, J. Rheol., 52(5): 1241-1268 (2008)

\section{Appendix A: Nomenclature}

$\begin{array}{ll}A & \text { Contact area }\left(m^{2}\right) \\ A_{h} & \text { Hamaker constant }(J) \\ C & \text { Solvation pressure constant }(M P a) \\ D_{i, j}^{k, l} & \text { Influence coefficient }(-) \\ E_{y} & \text { Barrier height for Eyring model }(J) \\ E & \text { Modulus of elasticity }(P a) \\ E^{\prime} & \text { Reduced modulus of elasticity }(\mathrm{Pa}) \\ F & \text { Friction force }(\mathrm{N}) \\ K & \text { Boltzman constant }\left(\mathrm{JK}^{-1}\right) \\ Q & \text { Process activation energy }(J) \\ T & \text { Temperature }\left({ }^{\circ} \mathrm{C}\right) \\ U & \text { Speed of entraining motion }(\mathrm{m} / \mathrm{s}) \\ V & \text { Speed of side-leakage }(\mathrm{m} / \mathrm{s}) \\ W & \text { Load carrying capacity }(\mathrm{N}) \\ Z & \text { Pressure-viscosity index }(-) \\ a & \text { Molecular diameter of lubricant }(\mathrm{m}) \\ g & \text { Switch function }(-)\end{array}$


$h \quad$ Gap size $(m)$

$h_{0} \quad$ Minimum rigid clearance $(m)$

$h_{s} \quad$ Local gap $(m)$

$i, k, x$ Coordinates along $x$-axis $(-)$

$j, l, y \quad$ Coordianates along $y$-axis $(-)$

$n \quad$ Iteration counter $(-)$

$p_{T} \quad$ Total pressure $(\mathrm{Pa})$

$p_{c} \quad$ Cavitation pressure $(P a)$

$p_{h} \quad$ Hydrodynamic pressure $(P a)$

$p_{s} \quad$ Solvation pressure $(\mathrm{Pa})$

$p_{v} \quad$ Constant pressure for varying velocities $(\mathrm{Pa})$

$p_{w} \quad$ van der Waals pressure $(P a)$

$r \quad$ Contact curvature radius $(m)$

$t \quad$ Time step $(s)$

$v_{0} \quad$ Characteristic velocity $(\mathrm{m} / \mathrm{s})$

$\Omega \quad$ Lubricant volume where pressure, $p_{T}$ is acting on $\left(m^{3}\right)$

$\alpha \quad$ Pressure-viscosity coefficient $\left(\mathrm{Pa}^{-1}\right)$

$\alpha_{o} \quad$ Viscosity coefficient at $p=0$ and $60^{\circ} \mathrm{C}\left(\mathrm{Pa}^{-1}\right)$

$\beta \quad$ Lubricant bulk modulus $(P a)$

$\delta \quad$ Elastic deformation $(m)$

$\epsilon_{\theta} \quad$ Convergence criteria for $\theta(-)$

$\eta \quad$ Lubricant viscosity (Pa.s)

$\eta_{0} \quad$ Lubricant viscosity at $p=0$ and $60^{\circ} \mathrm{C}$ (Pa.s)

$\nu \quad$ Poisson's ratio $(-)$

$\omega \quad$ Relaxation factor for $\theta(-)$

$\phi \quad$ Activation volume $\left(\mathrm{m}^{3}\right)$

$\rho \quad$ Lubricant density $\left(\mathrm{kg} / \mathrm{m}^{3}\right)$

$\rho_{c} \quad$ Lubricant density during cavitation $\left(\mathrm{kg} / \mathrm{m}^{3}\right)$

$\rho_{0} \quad$ Lubricant density at $p=0$ and constant temperature $\left(\mathrm{kg} / \mathrm{m}^{3}\right)$

$\tau \quad$ Shear stress $(P a)$

$\tau_{1} \quad$ Eyring shear stress (Velocity dependent) $(P a)$

$\tau_{0} \quad$ Eyring shear stress (Pressure dependent) $(\mathrm{Pa})$

$\theta \quad$ Non-dimensional density or Fractional film content $(-)$

$\xi \quad$ Eyring model constant (-) 